\title{
REPRESENTATION AND PREDICTION FOR LOCALLY HARMONIZABLE ISOTROPIC RANDOM FIELDS
}

\author{
RANDALL J. SWIFT \\ Western Kentucky University \\ Department of Mathematics \\ Bowling Green, KY 42101 USA
}

(Received November, 1994; Revised March, 1995)

\begin{abstract}
The class of harmonizable fields is a natural extension of the class of stationary fields. This paper considers fields whose increments are harmonizable and isotropic. Spectral representations are obtained for locally harmonizable isotropic fields. A linear least squares prediction for locally harmonizable isotropic fields is considered.
\end{abstract}

Key words: Weakly and Strongly Harmonizable Fields, Isotropic, Nonstationary, MT Integrals, Linear Prediction.

AMS (MOS) subject classifications:60G12, 60G35.

\section{Introduction}

The wide applications of stationary stochastic processes and random fields in the engineering and physical sciences has been well documented. The recent texts by Adler [1] and Yaglom [17], contain nice expositions of these applications. However, there are applications under which the assumption of stationarity is not physically realistic, e.g., detection of a phase modulated signal. Harmonizable processes provide a natural extension to the class of stationary processes. Harmonizable processes retain the powerful Fourier analytic techniques inherent with stationary processes while relaxing the assumption of stationarity. A detailed treatment is given in Rao [8].

Random fields admit a further property, termed isotropy. Isotropic random fields have certain characteristics invariant under rotation. In the recent works of Rao [10] and Swift [12], [13], the theory of harmonizable isotropic random fields is developed. A useful addition to this theory is given by considering a random field $X(t)$ which is not necessarily harmonizable, but whose increment field

$$
I_{\tau} X(t)=X(t+\tau)-X(t)
$$

is harmonizable. The theory of processes with harmonizable increments is developed in the paper by Swift [14]. Section 2 outlines the basic theory of harmonizable processes and fields. The spectral representations for the locally harmonizable isotropic random fields are obtained in sections 3 and 4. Section 4 also contains a construction of an example of a locally harmonizable isotropic random field. Section 5 develops a linear model for locally harmonizable isotropic fields. 


\section{Preliminaries}

To begin with, several ideas are briefly recalled here, which will be made use of later. First there is always an underlying probability space, $(\Omega, \Sigma, P)$.

In this paper, second order random fields are considered. More specifically, a map $X: I \rightarrow$ $L_{0}^{2}(P)$, where $I\left(\subset \mathbb{R}^{n}\right)$ an index set, and $L_{0}^{2}(P)$ is the space of all complex valued $f \in L_{0}^{2}$ such that $\int_{\Omega} f(\omega) d P(\omega)=0$.

A random field $X(\cdot)$ is termed stationary if its covariance function $r(\cdot, \cdot)$ is continuous and $r(s, t)=\tilde{r}(s-t)$. It can be represented as

$$
\tilde{r}(\boldsymbol{\tau})=\int_{\mathbb{R}^{n}} e^{i \boldsymbol{\lambda} \cdot \boldsymbol{\tau}} d \boldsymbol{F}(\boldsymbol{\lambda})
$$

for a unique non-negative bounded Borel measure $F(\cdot)$ on $\mathbb{R}^{n}$. The representation follows from a classical theorem of Bochner's [5] and is the motivation for the following definition.

Definition 2.1: A random field $X: \mathbb{R}^{n} \rightarrow L_{0}^{2}(P)$ is weakly harmonizable if its covariance $r(\cdot, \cdot)$ is expressible as

$$
r(\boldsymbol{s}, \boldsymbol{t})=\int_{\mathbb{R}^{n}} \int_{\mathbb{R}^{n}} e^{i \boldsymbol{\lambda} \cdot \boldsymbol{s}-i \boldsymbol{\lambda}^{\prime} \cdot \boldsymbol{t}} d F\left(\boldsymbol{\lambda}, \boldsymbol{\lambda}^{\prime}\right)
$$

where $F: \mathbb{R}^{n} \times \mathbb{R}^{n} \rightarrow \mathbb{C}$ is a positive semi-definite bimeasure, hence of finite Fréchet variation.

The integrals in (2) are strict Morse-Transue, [8]. A random field, $X(\cdot)$, is strongly harmonizable if the bi-measure $F(\cdot, \cdot)$ in (2) extends to a complex measure and hence is of bounded Vitali variation. In either case, $F(\cdot, \cdot)$ is termed the spectral bi-measure (or spectral measure) of the harmonizable field.

Comparison of equation (2) with equation (1) shows that when $F(\cdot, \cdot)$ concentrates on the diagonal $\boldsymbol{\lambda}=\boldsymbol{\lambda}^{\prime}$, both the weak and strong harmonizability concepts reduce to the stationary concept.

A subclass of stationary random fields satisfy an additional condition termed isotropy. Isotropic random fields $X(\cdot)$, can have covariance $\tilde{r}(\cdot)$ which are invariant under rotation and reflection in addition to translation. Isotropic fields play an important role in the statistical theory of turbulence, where direction in space is unimportant, Yaglom [18].

The representation of covariance $\tilde{r}(\cdot)$ for an isotropic homogeneous field was established by S. Bochner, [5] and is

$$
r(\boldsymbol{s}, \boldsymbol{t})=\tilde{r}(\boldsymbol{s}-\boldsymbol{t})=2^{\nu} \Gamma\left(\frac{n}{2}\right) \int_{0}^{\infty} \frac{J_{\nu}(\lambda \tau)}{(\lambda \tau)^{\nu}} d \tilde{F}(\lambda),
$$

where $\tau=\|\boldsymbol{s}-\boldsymbol{t}\|$. Here $J_{\nu}(\cdot)$ is the Bessel function (of the first kind) of order $\nu=\frac{n-2}{2}$ and $\widetilde{F}$ is a (unique) bounded Borel measure.

Following the idea in (2), a more general concept for isotropic random fields (which are not translation invariant) can be introduced, with the aid of strict Morse-Transue integration, Swift [12].

Definition 2.2: A random field $X: \mathbb{R} \rightarrow L_{0}^{2}(P)$ is weakly harmonizable isotropic if its covariance $r: \mathbb{R}^{n} \times \mathbb{R}^{n} \rightarrow \mathbb{C}$ is expressible as:

$$
r(\boldsymbol{s}, \boldsymbol{t})=2^{\nu} \Gamma\left(\frac{n}{2}\right) \int_{0}^{\infty} \int_{0}^{\infty} \frac{J_{\nu}\left(\left\|\lambda \boldsymbol{s}-\lambda^{\prime} \boldsymbol{t}\right\|\right)}{\left\|\lambda \boldsymbol{s}-\lambda^{\prime} \boldsymbol{t}\right\|^{\nu}} d F\left(\lambda, \lambda^{\prime}\right)
$$


where $\nu=\frac{n-2}{2}$ and $F(\cdot, \cdot)$ is a complex function of bounded Fréchet variation. (Here and in all such places later on, the integrals of (4) are understood in the strict Morse-Transue sense as in [2].)

If $F(\cdot, \cdot)$ concentrates on the diagonal $\lambda=\lambda^{\prime}$, so that $r(\boldsymbol{s}, \boldsymbol{t})=\tilde{r}(\boldsymbol{s}-\boldsymbol{t})$, equation (4) reduces to Bochner's representation (3). The integrals in (4) are strict Morse-Transue, [2]. As in definition 2.1 , a random field, $X(\cdot)$, is strongly harmonizable isotropic if the bimeasure $F(\cdot, \cdot)$ in $(4)$ extends to a complex measure and hence is of bounded Vitali variation. A detailed treatment of harmonizability can be found in Rao [8]. Harmonizable isotropic random fields are considered in extensive detail in Rao [10] and Swift [12], [13].

\section{Locally Harmonizable Isotropic Random Fields}

The concept of a locally harmonizable isotropic random field was introduced earlier and is formally given by

Definition 3.1: A random field $X: \mathbb{R}^{n} \rightarrow L_{0}^{2}(P)$ is locally weakly harmonizable if for each $\tau \in \mathbb{R}^{n}$, the increment field

$$
I_{\boldsymbol{\tau}} X(\boldsymbol{t})=X(\boldsymbol{t}+\boldsymbol{\tau})-X(\boldsymbol{t})
$$

is weakly harmonizable.

Locally weakly harmonizable random fields are also known as random fields with weakly harmonizable increments. Rao [9] considered the spectral representation and structure functions of these fields. Rao showed the representations are obtained by considering the concept of a generalized random field. For local processes and fields, generalized processes and fields are natural to consider since they provide the required differentiability concepts. The following is a brief outline of the development of generalized processes from Gelfand and Vilenkin [4] and Yaglom [17].

Consider the space $\mathfrak{F}$ of infinitely differentiable functions $h(t)$ having compact supports, which with compact convergence becomes a locally convex linear topological space. A generalized random field $X$ is a linear functional $X: \mathscr{K}_{G} \rightarrow \mathbb{C}$ such that if $\left\{\phi_{n}\right\}_{n=1}^{\infty} \subset \mathscr{K}, \phi_{n} \rightarrow 0$ in the topology of $\mathscr{K}$, then $X\left(\phi_{n}\right) \rightarrow 0$ in probability, as $n \rightarrow \infty$.

The mean of a generalized random field is the linear functional

$$
m(h)=E(X(h)), h \in \mathscr{K}
$$

and similarly its covariance is the bilinear functions $(m=0)$

$$
r\left(h_{1}, h_{2}\right)=E\left(X\left(h_{1}\right) \overline{\left.X\left(h_{2}\right)\right)}, h_{i} \in \mathscr{K}, \quad i=1,2 .\right.
$$

Ordinary fields generate the corresponding generalized fields by the relation

$$
X(h)=\int_{\mathbb{R}^{n}} X(\boldsymbol{t}) h(\boldsymbol{t}) d \boldsymbol{t} \text { for } h \in \mathscr{K} .
$$

The converse is not true unless an additional condition is assumed. That is, if a generalized field $X(\cdot)$ has point values (also called "of function space type") then the reverse implication holds. Using this, and results from the theory of generalized functions, one defines the derivative
$X^{\left(m_{1}, \ldots, m_{n}\right)}(h)$ of a generalized field $X(h)$ as 


$$
X^{\left(m_{1}, \ldots, m_{n}\right)}(h)=(-1)^{M} X\left(h^{\left(m_{1}, \ldots, m_{n}\right)}\right), \quad M=m_{1}+\ldots+m_{n}
$$

Then it follows that if $X(h)$ has point values $X(t)$ and if $X(t)$ is harmonizable then $X^{\left(m_{1}, \ldots, m_{n}\right)}(h)$ also has point values coinciding with $X^{\left(m_{1}, \ldots, m_{n}\right)}(t)$. Hence, an ordinary field $X(t)$ may be regarded as having a weak derivative, in the above sense, i.e., by allowing this derivative to be a generalized field. This condition is used to develop further the results on fields with harmonizable increments. A generalized field $X(\cdot)$ is weakly harmonizable if

$$
E(X(h))=0
$$

and $r(\cdot, \cdot)$ admits a representation

$$
r\left(h_{1}, h_{2}\right)=\int_{\mathbb{R}^{n}} \int_{\mathbb{R}^{n}} \widehat{h}_{1}(\boldsymbol{\lambda}) \overline{\hat{h}_{2}\left(\boldsymbol{\lambda}^{\prime}\right)} d F\left(\boldsymbol{\lambda}, \boldsymbol{\lambda}^{\prime}\right),
$$

where $F(\cdot, \cdot)$ is a positive definite function which defines a "tempered" bi-measure (measure in the strongly harmonizable case) with the integral interpreted as strict Morse-Transue in the weak case. It may be shown that such an $X(\cdot)$ admits a representation

$$
X(h)=\int_{\mathbb{R}^{n}} \widehat{h}(\boldsymbol{\lambda}) d Z(\boldsymbol{\lambda})
$$

where $Z: \mathfrak{B} \rightarrow L^{2}(P)$ is a vector measure such that

$$
E(Z(A) \overline{Z(B)})=\int_{A} \int_{B} d F\left(\lambda, \lambda^{\prime}\right) .
$$

(Rao [9], for the strongly harmonizable case, and the result extends to the weakly harmonizable case also.)

In the present development, the additional concept of isotropy for these fields is considered. For locally stationary isotropic random fields, Yaglom [18] proved the following:

Theorem 3.1: If $X: 96 \rightarrow L^{2}(P)$ is a generalized locally stationary isotropic random field then the mean functional $m(h)$ is zero and the covariance functional has representation

$$
\begin{aligned}
r\left(h_{1}, h_{2}\right)=2^{\nu} \Gamma\left(\frac{n}{2}\right) & \int_{+0}^{\infty} \int_{\mathbb{R}^{n}} \int_{\mathbb{R}^{n}} h_{1}(\boldsymbol{s}) \overline{h_{2}(\boldsymbol{t})} \frac{J_{\nu}(\lambda\|\boldsymbol{s}-\boldsymbol{t}\|)}{(\lambda\|\boldsymbol{s}-\boldsymbol{t}\|)^{\nu}} d \boldsymbol{s} d \boldsymbol{t} d \phi(\lambda) \\
& +a^{2} \int_{\mathbb{R}^{n}} \int_{\mathbb{R}^{n}} \boldsymbol{s} \cdot \boldsymbol{t} h_{1}(\boldsymbol{s}) \overline{h_{2}(\boldsymbol{t})} d \boldsymbol{s} d \boldsymbol{t}
\end{aligned}
$$

where $\phi(\cdot)$ is a nondecreasing function which satisfies

$$
\int_{+0}^{\infty} \frac{\lambda^{2} d \phi(\lambda)}{\left(1+\lambda^{2}\right)^{p+1}}<\infty
$$

and $a$ is a non-negative constant, and $p>0$.

Theorem 3.1 motivates, (in the same fashion as Bochner's theorem motivated definition 2.1), the following definition: 
Definition 3.2: A generalized random field $X: \mathscr{G} \rightarrow L^{2}(P)$ is locally weakly harmonizable isotropic if it has mean function $m(h)$ zero, and a covariance functional representable as

$$
\begin{aligned}
r\left(h_{1}, h_{2}\right)=2^{\nu} \Gamma\left(\frac{n}{2}\right) \int_{+0}^{\infty} & \int_{+0}^{\infty} \int_{\mathbb{R}^{n}} \int_{\mathbb{R}^{n}} h_{1}(\boldsymbol{s}) \overline{h_{2}(\boldsymbol{t})} \frac{J_{\nu}\left(\left\|\lambda \boldsymbol{s}-\lambda^{\prime} \boldsymbol{t}\right\|\right)}{\left(\left\|\lambda \boldsymbol{s}-\lambda^{\prime} \boldsymbol{t}\right\|\right)^{\nu}} d \boldsymbol{s} d \boldsymbol{t} d \boldsymbol{F}\left(\lambda, \lambda^{\prime}\right) \\
& +a^{2} \int_{\mathbb{R}^{n}} \int_{\mathbb{R}^{n}} \boldsymbol{s} \cdot \boldsymbol{t} h_{1}(\boldsymbol{s}) \overline{h_{2}(\boldsymbol{t})} d \boldsymbol{s} d \boldsymbol{t}
\end{aligned}
$$

where $F(\cdot, \cdot)$ is a function of locally bounded Fréchet variation satisfying

$$
\int_{\mathbb{R}-\{0\}} \int_{\mathbb{R}-\{0\}\left(1+\lambda^{2} 1+\left(\lambda^{\prime}\right)^{2}\right)^{\frac{p+1}{2}}}^{\lambda \lambda^{\prime} d F\left(\lambda, \lambda^{\prime}\right)} \mid<\infty
$$

and $a$ is a non-negative constant, $p>0$, the integrals relative to $F$ being in the strict MT sense for the weak harmonizable as usual.

Spectral bi-measures $F(\cdot, \cdot)$ which satisfy equation (8) are known as tempered. Observe that if $F(\cdot, \cdot)$ concentrates on the diagonal $\lambda=\lambda^{\prime}$, equation (7) reduces to equation (6), so the locally stationary isotropic concept is subsumed in this definition. The following theorem is a useful characterization of the covariance functionals of generalized locally harmonizable isotropic fields. The proof of which depends upon the following lemma:

Lemma 3.1: With the above notation, one has

$$
\alpha_{n}^{2} \sum_{m=0}^{\infty} \sum_{l=1}^{h(m, n)} S_{m}^{l}(\boldsymbol{u}) S_{m}^{l}(\boldsymbol{v}) \frac{J_{m+\nu}\left(\lambda r_{1}\right) J_{m+\nu}\left(\lambda^{\prime} r_{2}\right)}{\left(\lambda r_{1}\right)^{\nu}\left(\lambda^{\prime} r_{2}\right)^{\nu}}=2^{\nu} \Gamma\left(\frac{n}{2}\right) \frac{J_{\nu}\left(\lambda R\left(\lambda, \lambda^{\prime}\right)\right)}{\left(\lambda R\left(\lambda, \lambda^{\prime}\right)\right)^{\nu}}
$$

where $R\left(\lambda, \lambda^{\prime}\right)=\left(r_{1}^{2}+\left(\frac{\lambda^{\prime}}{\lambda}\right)^{2} r_{2}^{2}-2\left(\frac{\lambda^{\prime}}{\lambda}\right) r_{1} r_{2} \cos \theta\right)^{\frac{1}{2}}$ and $\cos \theta=\langle\boldsymbol{u}, \boldsymbol{v}\rangle$.

The proof of this lemma follows from standard arguments and may be found in Swift [12].

Theorem 3.2: A generalized random field $X: \mathfrak{G} \rightarrow L^{2}(P)$ is locally weakly harmonizable isotropic if and only if it covariance functional is representable as:

$$
\begin{gathered}
r\left(h_{1}, h_{2}\right)=\alpha_{n}^{2} \int_{\mathbb{R}^{n}} \int_{\mathbb{R}^{n}} \int_{+0}^{\infty} \int_{+0}^{\infty} \sum_{m=0}^{\infty} \sum_{l=1}^{h(m, n)} h_{1}(\boldsymbol{s}) \overline{h_{2}(\boldsymbol{t})} \\
\times S_{m}^{l}(\boldsymbol{u}) S_{m}^{l}(\boldsymbol{v}) \frac{J_{m+\nu}(\lambda\|\boldsymbol{s}\|) J_{m+\nu}\left(\lambda^{\prime}\|\boldsymbol{t}\|\right)}{(\lambda\|\boldsymbol{s}\|)^{\nu}\left(\lambda^{\prime}\|\boldsymbol{t}\|\right)^{\nu}} d F\left(\lambda, \lambda^{\prime}\right) d \boldsymbol{s} d \boldsymbol{t} \\
+a \int_{\mathbb{R}^{n}} \int_{\mathbb{R}^{n}} \boldsymbol{s} \cdot \boldsymbol{t} h_{1}(\boldsymbol{s}) \overline{h_{2}(\boldsymbol{t})} d \boldsymbol{s} d \boldsymbol{t}
\end{gathered}
$$

where $F(\cdot, \cdot)$ is a tempered function of locally bounded Fréchet variation and a is a non-negative constant.

Proof: In one direction, if $X(\cdot)$ is a generalized weakly harmonizable isotropic random field, the definition implies

$$
r\left(h_{1}, h_{2}\right)=2^{\nu} \Gamma\left(\frac{n}{2}\right) \int_{+0}^{\infty} \int_{+0}^{\infty} \int_{\mathbb{R}^{n}} \int_{\mathbb{R}^{n}} h_{1}(\boldsymbol{s}) \overline{h_{2}(\boldsymbol{t})} \frac{J_{\nu}\left(\left\|\lambda \boldsymbol{s}-\lambda^{\prime} \boldsymbol{t}\right\|\right)}{\left(\left\|\lambda \boldsymbol{s}-\lambda^{\prime} \boldsymbol{t}\right\|\right)^{\nu}} d \boldsymbol{s} d \boldsymbol{t} d F\left(\lambda, \lambda^{\prime}\right)
$$


But, by the law of cosines

$$
+a^{2} \int_{\mathbb{R}^{n}} \int_{\mathbb{R}^{n}} s \cdot t h_{1}(s) \overline{h_{2}(t)} d s d t .
$$

$$
\left\|\lambda \boldsymbol{s}-\lambda^{\prime} \boldsymbol{t}\right\|=\left(\|\lambda \boldsymbol{s}\|^{2}+\left\|\lambda^{\prime} \boldsymbol{t}\right\|^{2}-2\left\langle\lambda \boldsymbol{s}, \lambda^{\prime} \boldsymbol{t}\right\rangle\right)^{\frac{1}{2}}
$$

so that by lemma 3.1

$$
\begin{gathered}
r\left(h_{1}, h_{2}\right)=a_{n}^{2} \int_{\mathbb{R}^{n}} \int_{\mathbb{R}^{n}} h_{1}(\boldsymbol{s}) \overline{h_{2}(\boldsymbol{t})} \sum_{m=0}^{\infty} \sum_{l=1}^{h(m, n)} S_{m}^{l}(\boldsymbol{u}) S_{m}^{l}(\boldsymbol{v}) \\
\times \int_{+0}^{\infty} \int_{+0}^{\infty} \frac{J_{m+\nu}(\lambda\|\boldsymbol{s}\|) J_{m+\nu}\left(\lambda^{\prime}\|\boldsymbol{t}\|\right)}{(\lambda\|\boldsymbol{s}\|)^{\nu}\left(\lambda^{\prime}\|\boldsymbol{t}\|\right)^{\nu}} d F\left(\lambda, \lambda^{\prime}\right) d \boldsymbol{s} d \boldsymbol{t} \\
+a \int_{\mathbb{R}^{n}} \int_{\mathbb{R}^{n}} \boldsymbol{s} \cdot \boldsymbol{t} h_{1}(\boldsymbol{s}) \overline{h_{2}(\boldsymbol{t})} d \boldsymbol{s} d \boldsymbol{t}
\end{gathered}
$$

since the series is absolutely convergent and the interchange of the integrals and summations is justified. This establishes one implication. For the converse, it is only necessary to reverse the above steps. This completes the proof of the theorem.

This theorem will be used to obtain the spectral representations of the generalized and ordinary fields, as well as for the increment field.

\section{Spectral Representations}

Theorem 4.1: A generalized random field $X: \mathscr{H} \rightarrow L^{2}(P)$ is locally weakly harmonizable isotropic if and only if it has a spectral representation

$$
\begin{gathered}
X(h)=\alpha_{n} \int_{\mathbb{R}^{n}} h(t) \sum_{m=0}^{\infty} \sum_{l=1}^{h(m, n)} S_{m}^{l}(\boldsymbol{u}) \\
\times \int_{+0}^{\infty} \frac{J_{m+\nu}(\lambda\|\boldsymbol{t}\|)}{(\lambda\|\boldsymbol{t}\|)^{\nu}} d Z_{m}^{l}(\lambda) d \boldsymbol{t}+\boldsymbol{X}_{1} \cdot \int_{\mathbb{R}^{n}} \boldsymbol{t} h(\boldsymbol{t}) d \boldsymbol{t}
\end{gathered}
$$

where $E\left(Z_{m}^{l}\left(B_{1}\right) Z_{m^{\prime}}^{l^{\prime}}\left(B_{2}\right)\right)=\delta_{m m^{\prime}} \delta_{l l^{\prime}} F\left(B_{1}, B_{2}\right)$, with $F(\cdot, \cdot)$ a tempered function of locally bounded Fréchet variation, and $\boldsymbol{X}_{1}=\left(X_{11}, X_{12}, \ldots, X_{1 n}\right)$ is a random vector which satisfies

$$
E\left(X_{1 k} Z_{m}^{l}(B)\right)=0, \quad k=1, \ldots, n
$$

and

$$
E\left(X_{1 k} \overline{X_{1 j}}\right)= \begin{cases}0 & \text { for } k \neq j \\ a & \text { for } k=j .\end{cases}
$$

Proof: Suppose $x(\cdot)$ is a generalized locally harmonizable isotropic random field, then by theorem 3.2, the covariance functional is expressible as equation (10), 


$$
\begin{gathered}
r\left(h_{1}, h_{2}\right)=\alpha_{n}^{2} \int_{\mathbb{R}^{n}} \int_{\mathbb{R}^{n}} h_{1}(\boldsymbol{s}) \overline{h_{2}(\boldsymbol{t})} \sum_{m=0}^{\infty} \sum_{l=1}^{h(m, n)} S_{m}^{l}(\boldsymbol{u}) S_{m}^{l}(\boldsymbol{v}) \\
\times \int_{+0}^{\infty} \int_{+0}^{\infty} \frac{J_{\mu+\nu}(\lambda\|\boldsymbol{s}\|) J_{m+\nu}\left(\lambda^{\prime}\|\boldsymbol{t}\|\right)}{(\lambda\|\boldsymbol{s}\|)^{\nu}\left(\lambda^{\prime}\|\boldsymbol{t}\|\right)^{\nu}} d F\left(\lambda, \lambda^{\prime}\right) d \boldsymbol{s} d \boldsymbol{t} \\
+a \int_{\mathbb{R}^{n}} \int_{\mathbb{R}^{n}} \boldsymbol{s} \cdot \boldsymbol{t} h_{1}(\boldsymbol{s}) \overline{h_{2}(\boldsymbol{t})} d \boldsymbol{s} d \boldsymbol{t}
\end{gathered}
$$

where the series converges absolutely. Using the classical Karhunen type theorem, cf. Yaglom [18] for such a series, the spectral representation is obtained as

$$
\begin{gathered}
X(h)=\alpha_{n} \int_{\mathbb{R}^{n}} h(\boldsymbol{t}) \sum_{m=0}^{\infty} \sum_{l=1}^{h(m, n)} S_{m}^{l}(\boldsymbol{u}) \\
\times \int_{+0}^{\infty} \frac{J_{m+\nu}(\lambda\|\boldsymbol{t}\|)}{(\lambda\|\boldsymbol{t}\|)^{\nu}} d Z_{m}^{l}(\lambda) d \boldsymbol{t}+\boldsymbol{X}_{1} \cdot \int_{\mathbb{R}^{n}} \boldsymbol{t} h(\boldsymbol{t}) d \boldsymbol{t}
\end{gathered}
$$

where $\boldsymbol{X}_{1}=\left(X_{11}, X_{12}, \ldots, X_{1 n}\right)$ is a random vector which satisfies

$$
E\left(X_{1 k} Z_{m}^{l}(B)\right)=0, \quad k=1, \ldots, n
$$

and

$$
E\left(X_{1 k}, \overline{X_{1 j}}\right)= \begin{cases}0 & \text { for } k \neq j \\ a & \text { for } k=j\end{cases}
$$

The converse statement follows directly from the definition of a covariance and applying theorem 3.2 . The assertion is complete.

Using this theorem, the spectral representations of the field $X(\cdot)$, the increment field $I_{\boldsymbol{\tau}} X(\cdot)$, and the structure function $B(\cdot, \cdot, \cdot, \cdot)$ may now be obtained.

Theorem 4.2: A locally weakly harmonizable isotropic random field $X: \mathbb{R}^{n} \rightarrow L_{0}^{2}(P)$ has spectral representation

$$
X(\boldsymbol{t})=\alpha_{n} \sum_{m=0}^{\infty} \quad \sum_{l=1}^{h(m, n)} S_{m}^{1}(\boldsymbol{u}) \int_{+0}^{\infty} \frac{J_{m+\nu}(\lambda\|\boldsymbol{t}\|)}{(\lambda\|\boldsymbol{t}\|)^{\nu}} d Z_{m}^{l}(\lambda)+\boldsymbol{X}_{1} \cdot \boldsymbol{t}
$$

and the increment field $\boldsymbol{I}_{\boldsymbol{\tau}} X(\cdot)$ has the structure function

$$
\begin{gathered}
B\left(\boldsymbol{s}, \boldsymbol{t}, \boldsymbol{s}+\boldsymbol{\tau}_{1}, \boldsymbol{t}+\boldsymbol{\tau}_{2}\right)=2^{\nu} \Gamma\left(\frac{n}{2}\right) \int_{+0}^{\infty} \int_{+0}^{\infty}\left(\frac{J_{\nu}\left(\left\|\lambda\left(\boldsymbol{s}+\boldsymbol{\tau}_{1}\right)-\lambda^{\prime}\left(\boldsymbol{t}+\boldsymbol{\tau}_{2}\right)\right\|\right)}{\left\|\lambda\left(\boldsymbol{s}+\boldsymbol{\tau}_{1}\right)-\lambda^{\prime}\left(\boldsymbol{t}+\boldsymbol{\tau}_{2}\right)\right\|^{\nu}}\right. \\
-\frac{J_{\nu}\left(\left\|\lambda\left(\boldsymbol{s}+\boldsymbol{\tau}_{1}\right)-\lambda^{\prime} \boldsymbol{t}\right\|\right)}{\left\|\lambda\left(\boldsymbol{s}+\boldsymbol{\tau}_{1}\right)-\lambda^{\prime} \boldsymbol{t}\right\|^{\nu}}-\frac{J_{\nu}\left(\| \lambda \boldsymbol{s}-\lambda^{\prime}\left(\boldsymbol{t}+\boldsymbol{\tau}_{2}\right)\right.}{\left\|\lambda \boldsymbol{s}-\lambda^{\prime}\left(\boldsymbol{t}+\boldsymbol{\tau}_{2}\right)\right\|^{\nu}} \\
\left.+\frac{J_{\nu}\left(\left\|\lambda \boldsymbol{s}-\lambda^{\prime} \boldsymbol{t}\right\|\right)}{\left\|\lambda \boldsymbol{s}-\lambda^{\prime} \boldsymbol{t}\right\|^{\nu}}\right) d F\left(\lambda, \lambda^{\prime}\right)+a \boldsymbol{\tau}_{1} \cdot \boldsymbol{\tau}_{2},
\end{gathered}
$$


where $F(\cdot, \cdot)$ is a tempered function of bounded Vitali variation, related to the $Z_{m}^{l}(\cdot)$-measure, and $\boldsymbol{X}_{1}=\left(X_{11}, X_{12}, \ldots, X_{1 n}\right)$ is a random vector which satisfies

$$
E\left(X_{1 k} Z_{m}^{l}(B)\right)=0, \quad k=1, \ldots, n, \quad \forall B \in \Sigma
$$

and

$$
E\left(X_{1 k}, \overline{X_{1 j}}\right)= \begin{cases}0 & \text { for } k \neq j \\ a & \text { for } k=j\end{cases}
$$

and a, a nonnegative constant.

Proof: Using the relationship

$$
X(h)=\int_{\mathbb{R}^{n}} h(\boldsymbol{t}) X(\boldsymbol{t}) d \boldsymbol{t}
$$

with the spectral representation of the previous theorem one has (since $X(\cdot)$ is point valued),

$$
X(\boldsymbol{t})=\alpha_{n} \sum_{m=0}^{\infty} \sum_{l=1}^{h(m, n)} S_{m}^{l}(\boldsymbol{u}) \int_{+0}^{\infty} \frac{J_{m+\nu}(\lambda\|\boldsymbol{t}\|)}{(\lambda\|\boldsymbol{t}\|)^{\nu}} d Z_{m}^{l}(\lambda)+\boldsymbol{X}_{1} \cdot \boldsymbol{t} .
$$

This representation gives the increment field,

$$
\begin{aligned}
\boldsymbol{I}_{\boldsymbol{\tau}} X(\boldsymbol{t})= & \alpha_{n} \sum_{m=0}^{\infty} \sum_{l=1}^{h(m, n)}\left(S_{m}^{l}\left(\boldsymbol{u}_{1}\right) \int_{+0}^{\infty} \frac{J_{m+\nu}(\lambda\|\boldsymbol{t}+\boldsymbol{\tau}\|)}{(\lambda \| \boldsymbol{t}+\boldsymbol{\tau})^{\nu}} d Z_{m}^{l}(\lambda)\right. \\
& \left.-S_{m}^{l}\left(\boldsymbol{u}_{2}\right) \int_{+0}^{\infty} \frac{J_{m+\nu}(\lambda\|\boldsymbol{t}\|)}{(\lambda\|\boldsymbol{t}\|)^{\nu}} d Z_{m}^{l}(\lambda)\right)+\boldsymbol{X}_{1} \cdot \boldsymbol{\tau} .
\end{aligned}
$$

Now since $E\left(Z_{m}^{l}(\lambda)\right)=0$, the structure function is

$$
\begin{gathered}
B\left(\boldsymbol{s}, \boldsymbol{t}, \boldsymbol{s}+\boldsymbol{\tau}_{1}, \boldsymbol{t}+\boldsymbol{\tau}_{2}\right)=E\left(\boldsymbol{I}_{\boldsymbol{\tau}_{1}} X(\boldsymbol{s}) \overline{\boldsymbol{I}_{\tau_{2}} X(\boldsymbol{t})}\right) \\
=\alpha_{n=0}^{2} \sum_{m=1}^{\infty} \sum_{l=1}^{h(m, n)} S_{m}^{l}\left(\boldsymbol{u}_{1}\right) S_{m}^{l}\left(\boldsymbol{v}_{1}\right) \int_{+0}^{\infty} \int_{+0}^{\infty} \frac{J_{m+\nu}\left(\lambda\left\|\boldsymbol{s}+\boldsymbol{\tau}_{1}\right\|\right) J_{m+\nu}\left(\lambda^{\prime}\left\|\boldsymbol{t}+\boldsymbol{\tau}_{2}\right\|\right)}{\left(\lambda\left\|\boldsymbol{s}+\boldsymbol{\tau}_{1}\right\|\right)^{\nu}\left(\lambda^{\prime}\left\|\boldsymbol{t}+\boldsymbol{\tau}_{2}\right\|\right)^{\nu}} d F\left(\lambda, \lambda^{\prime}\right) \\
-\alpha_{n}^{2} \sum_{m=0}^{\infty} \sum_{l=1}^{h(m, n)} S_{m}^{l}\left(\boldsymbol{u}_{1}\right) S_{m}^{l}\left(\boldsymbol{v}_{2}\right) \int_{+0}^{\infty} \int_{+0}^{\infty} \frac{J_{m+\nu}\left(\lambda\left\|\boldsymbol{s}+\boldsymbol{\tau}_{1}\right\|\right) J_{m+\nu}\left(\lambda^{\prime}\|\boldsymbol{t}\|\right)}{\left(\lambda\left\|\boldsymbol{s}+\boldsymbol{\tau}_{1}\right\|\right)^{\nu}\left(\lambda^{\prime}\|\boldsymbol{t}\|\right)^{\nu}} d F\left(\lambda, \lambda^{\prime}\right) \\
-\alpha_{n}^{2} \sum_{m=0}^{\infty} \sum_{l=1}^{h(m, n)} S_{m}^{l}\left(\boldsymbol{u}_{2}\right) S_{m}^{l}\left(\boldsymbol{v}_{1}\right) \int_{+0}^{\infty} \int_{+0}^{\infty} \frac{J_{m+\nu}(\lambda\|\boldsymbol{s}\|) J_{m+\nu}\left(\lambda^{\prime}\left\|\boldsymbol{t}+\boldsymbol{\tau}_{2}\right\|\right)}{(\lambda\|\boldsymbol{s}\|)^{\nu}\left(\lambda^{\prime}\left\|\boldsymbol{t}+\boldsymbol{\tau}_{2}\right\|\right)^{\nu}} d F\left(\lambda, \lambda^{\prime}\right) \\
+\alpha_{n}^{2} \sum_{m=0}^{\infty} \sum_{l=1}^{h(m, n)} S_{m}^{l}\left(\boldsymbol{u}_{2}\right) S_{m}^{l}\left(\boldsymbol{v}_{2}\right) \int_{+0}^{\infty} \int_{+0}^{\infty} \frac{J_{m+\nu}(\lambda\|\boldsymbol{s}\|) J_{m+\nu}\left(\lambda^{\prime}\|\boldsymbol{t}\|\right)}{(\lambda\|\boldsymbol{s}\|)^{\nu}\left(\lambda^{\prime}\|\boldsymbol{t}\|\right)^{\nu}} d F\left(\lambda, \lambda^{\prime}\right)
\end{gathered}
$$




$$
+a \tau_{1} \cdot \tau_{2}
$$

Hence, using lemma 3.1, equation (15) becomes

$$
\begin{gathered}
B\left(\boldsymbol{s}, \boldsymbol{t}, \boldsymbol{s}+\boldsymbol{\tau}_{1}, \boldsymbol{t}+\boldsymbol{\tau}_{2}\right)=2^{\nu} \Gamma\left(\frac{n}{2}\right) \int_{+0}^{\infty} \int_{+0}^{\infty} \frac{J_{\nu}\left(\left\|\lambda\left(\boldsymbol{s}+\boldsymbol{\tau}_{1}\right)-\lambda^{\prime}\left(\boldsymbol{t}+\boldsymbol{\tau}_{2}\right)\right\|\right)}{\left\|\lambda\left(\boldsymbol{s}+\boldsymbol{\tau}_{1}\right)-\lambda^{\prime}\left(\boldsymbol{t}+\boldsymbol{\tau}_{2}\right)\right\|^{\nu}} d F\left(\lambda, \lambda^{\prime}\right) \\
-2^{\nu} \Gamma\left(\frac{n}{2}\right) \int_{+0}^{\infty} \int_{+0}^{\infty} \frac{J_{\nu}\left(\left\|\lambda\left(\boldsymbol{s}+\boldsymbol{\tau}_{1}\right)-\lambda^{\prime} \boldsymbol{t}\right\|\right)}{\left\|\lambda\left(\boldsymbol{s}+\boldsymbol{\tau}_{1}\right)-\lambda^{\prime} \boldsymbol{t}\right\|^{\nu}} d F\left(\lambda, \lambda^{\prime}\right) \\
-2^{\nu} \Gamma\left(\frac{n}{2}\right) \int_{+0}^{\infty} \int_{+0}^{\infty} \frac{J_{\nu}\left(\left\|\lambda \boldsymbol{s}-\lambda^{\prime}\left(\boldsymbol{t}+\boldsymbol{\tau}_{2}\right)\right\|\right)}{\left\|\lambda \boldsymbol{s}-\lambda^{\prime}\left(\boldsymbol{t}+\boldsymbol{\tau}_{2}\right)\right\|^{\nu}} d F\left(\lambda, \lambda^{\prime}\right) \\
+2^{\nu} \Gamma\left(\frac{n}{2}\right) \int_{+0}^{\infty} \int_{+0}^{\infty} \frac{J_{\nu}\left(\left\|\lambda \boldsymbol{s}-\lambda^{\prime} \boldsymbol{t}\right\|\right)}{\left\|\lambda \boldsymbol{s}-\lambda^{\prime} \boldsymbol{t}\right\|^{\nu}} d F\left(\lambda, \lambda^{\prime}\right)+a \boldsymbol{\tau}_{1} \cdot \boldsymbol{\tau}_{2} .
\end{gathered}
$$

Thus, writing equation (16) as one integral

$$
\begin{gathered}
B\left(s, \boldsymbol{t}, \boldsymbol{s}+\boldsymbol{\tau}_{1}, \boldsymbol{t}+\boldsymbol{\tau}_{2}\right)=2^{\nu} \Gamma\left(\frac{n}{2}\right) \int_{+0}^{\infty} \int_{+0}^{\infty}\left(\frac{J_{\nu}\left(\left\|\lambda\left(\boldsymbol{s}+\boldsymbol{\tau}_{1}\right)-\lambda^{\prime}\left(\boldsymbol{t}+\boldsymbol{\tau}_{2}\right)\right\|\right)}{\left\|\lambda\left(\boldsymbol{s}+\boldsymbol{\tau}_{1}\right)+\lambda^{\prime}\left(\boldsymbol{t}-\boldsymbol{\tau}_{2}\right)\right\|^{\nu}}\right. \\
-\frac{J_{\nu}\left(\left\|\lambda\left(s+\boldsymbol{\tau}_{1}\right)-\lambda^{\prime} \boldsymbol{t}\right\|\right)}{\left\|\lambda\left(\boldsymbol{s}+\boldsymbol{\tau}_{1}\right)+\lambda^{\prime} \boldsymbol{t}\right\|^{\nu}}-\frac{J_{\nu}\left(\left\|\lambda \boldsymbol{s}-\lambda^{\prime}\left(\boldsymbol{t}-\boldsymbol{\tau}_{2}\right)\right\|\right)}{\left\|\lambda \boldsymbol{s}+\lambda^{\prime}\left(\boldsymbol{t}-\boldsymbol{\tau}_{2}\right)\right\|^{\nu}} \\
\left.+\frac{J_{\nu}\left(\left\|\lambda \boldsymbol{s}-\lambda^{\prime} \boldsymbol{t}\right\|\right)}{\left\|\lambda \boldsymbol{s}-\lambda^{\prime} \boldsymbol{t}\right\|^{\nu}}\right) d F\left(\lambda, \lambda^{\prime}\right)+a \tau_{1} \cdot \tau_{2}
\end{gathered}
$$

which completes the proof.

The proof of this theorem contained a useful fact about $B(\cdot, \cdot, \cdot, \cdot)$ stated here as corollary for later reference.

Corollary 4.1: The structure function $B(\cdot, \cdot, \cdot, \cdot)$ of a locally strongly harmonizable isotropic random field has a spherical polar representation as equation (15).

Consider the form of equation (13) for $s=t$ and $\tau_{1}=\tau_{2}=\tau$, that is

$$
\begin{gathered}
B(\boldsymbol{t}, \boldsymbol{t}, \boldsymbol{t}+\boldsymbol{\tau}, \boldsymbol{t}+\boldsymbol{\tau})=2^{\nu} \Gamma\left(\frac{n}{2}\right) \int_{+0}^{\infty} \int_{+0}^{\infty}\left(\frac{J_{\nu}\left(\left\|\left(\lambda-\lambda^{\prime}\right)(\boldsymbol{t}+\boldsymbol{\tau})\right\|\right)}{\left\|\left(\lambda-\lambda^{\prime}\right)\left(\boldsymbol{t}+\boldsymbol{\tau}_{2}\right)\right\|^{\nu}}\right. \\
-\frac{J_{\nu}\left(\left\|\left(\lambda-\lambda^{\prime}\right) \boldsymbol{t}+\lambda \boldsymbol{\tau}\right\|\right)}{\left\|\left(\lambda-\lambda^{\prime}\right) \boldsymbol{t}+\lambda \boldsymbol{\tau}\right\|^{\nu}}-\frac{J_{\nu}\left(\left\|\left(\lambda-\lambda^{\prime}\right) \boldsymbol{t}-\lambda^{\prime} \boldsymbol{\tau}_{2}\right\|\right)}{\left\|\left(\lambda-\lambda^{\prime}\right) \boldsymbol{t}-\lambda^{\prime} \boldsymbol{\tau}_{2}\right\|^{\nu}} \\
\left.+\frac{J_{\nu}\left(\left\|\left(\lambda-\lambda^{\prime}\right) \boldsymbol{t}\right\|\right)}{\left\|\left(\lambda-\lambda^{\prime}\right) \boldsymbol{t}\right\|^{\nu}}\right) d F\left(\lambda, \lambda^{\prime}\right)+a \boldsymbol{\tau} \cdot \boldsymbol{\tau} .
\end{gathered}
$$


Observe here that if $F(\cdot, \cdot)$ concentrates on the diagonal $\lambda=\lambda^{\prime}$, using

$$
\lim _{x \rightarrow 0} \frac{J_{\nu}(x)}{x^{\nu}}=\frac{1}{2^{\nu} \Gamma\left(\frac{n}{2}\right)}
$$

equation (17) becomes

$$
\begin{gathered}
B(\boldsymbol{t}, \boldsymbol{t}, \boldsymbol{t}+\boldsymbol{\tau}, \boldsymbol{t}+\boldsymbol{\tau})=(D(\|\boldsymbol{\tau}\|), \text { say }) \\
=2 \int_{+0}^{\infty}\left(1-2^{\nu} \Gamma\left(\frac{n}{2}\right) \frac{J_{\nu}(\lambda\|\boldsymbol{\tau}\|)}{\left(\lambda\|\tau\|^{\nu}\right.}\right) d \tilde{F}(\lambda)+a \boldsymbol{\tau} \cdot \boldsymbol{\tau}
\end{gathered}
$$

which is the form obtained by Yaglom ([17], pp. 310), for the structure function of a locally statationary isotropic random field. Thus equation (17) is an extension to the locally stationary case.

As was done for harmonizable isotropic fields (see Swift [12]), it is possible to obtain the spectral representation of a locally isotropic random field which is not necessarily locally harmonizable.

Theorem 4.3: A locally isotropic random field $X: \mathbb{R}^{n} \rightarrow L_{0}^{2}(P)$ has spectral representation

$$
X(\boldsymbol{t})=\sum_{m=0}^{\infty} \sum_{l=1}^{h(m, n)} S_{m}^{l}(\boldsymbol{u}) Y_{m}^{l}(\|\boldsymbol{t}\|)+\boldsymbol{X}_{1} \cdot \boldsymbol{t},
$$

and the increment field, $\boldsymbol{I}_{\boldsymbol{\tau}} X(\cdot)$, has the structure function

$$
\begin{aligned}
B\left(\boldsymbol{s}, \boldsymbol{t}, \boldsymbol{s}+\boldsymbol{\tau}_{1}, \boldsymbol{t}+\boldsymbol{\tau}_{2}\right)= & \frac{1}{\omega_{n}} \sum_{m=0}^{\infty} \frac{h(m, n)}{C_{m}^{n}(1)}\left(C_{m}^{n}\left(\cos \theta_{1}\right) b_{m}\left(\left\|\boldsymbol{s}+\boldsymbol{\tau}_{1}\right\|,\left\|\boldsymbol{t}+\boldsymbol{\tau}_{2}\right\|\right)\right. \\
& -C_{m}^{n}\left(\cos \theta_{2}\right) b_{m}\left(\left\|\boldsymbol{s}+\boldsymbol{\tau}_{1}\right\|,\|\boldsymbol{t}\|\right) \\
& -C_{m}^{n}\left(\cos \theta_{3}\right) b_{m}\left(\|\boldsymbol{s}\|,\left\|\boldsymbol{t}+\boldsymbol{\tau}_{2}\right\|\right) \\
+ & C_{m}^{n}\left(\cos \theta_{4}\right) b_{m}(\|\boldsymbol{s}\|,\|\boldsymbol{t}\|)+a \boldsymbol{\tau}_{1} \cdot \boldsymbol{\tau}_{2}
\end{aligned}
$$

where $Y_{m}^{l}(\cdot)$ is a sequence of stochastic processes which satisfy

$$
E\left(Y_{m}^{l}(s) \overline{Y_{m^{\prime}}^{l^{\prime}}(t)}\right)=\delta_{m m^{\prime}} \delta_{l l^{\prime}} b_{m}(s, t)
$$

with $b_{m}(\cdot, \cdot)$ satisfying

$$
\sum_{m=0}^{\infty} h(m, n) b_{m}\left(\tau_{1}, \tau_{2}\right)<\infty
$$

and

$$
b_{m}(0, \tau)=0 \text { for } m \neq 0 .
$$

Here $C_{m}^{\nu}(\cdot)$ are the ultraspherical polynomials. Further, $\boldsymbol{X}_{1}$ a fixed random vector satisfying

$$
E\left(X_{1 k} Z_{m}^{l}(B)\right)=0, \quad k=1, \ldots, n
$$

and 


$$
E\left(X_{1 k} \overline{X_{1 j}}\right)= \begin{cases}0 & \text { for } j \neq j \\ a & \text { for } k=j\end{cases}
$$

$\theta_{k}, k=1, \ldots, 4$ the angles between the pairs of vectors $\boldsymbol{s}+\boldsymbol{\tau}_{1}, \boldsymbol{t}+\boldsymbol{\tau}_{2}, \ldots, \boldsymbol{s}, \boldsymbol{t}$, and a, a non-negative constant.

Proof: Letting

$$
Y_{m}^{l}(r)=\alpha_{n} \int_{0}^{\infty} \frac{J_{m+\nu}(\lambda r)}{(\lambda r)^{\nu}} d Z_{m}^{l}(\lambda)
$$

one has

$$
E\left(Y_{m}^{l}(r)\right)=0
$$

and

$$
E\left(Y_{m}^{l}\left(r_{1}\right) \overline{\left.Y_{m^{\prime}}^{l^{\prime}}\left(r_{2}\right)\right)}=\delta_{m m^{\prime}} \delta_{l l^{\prime}} F\left(r_{1}, r_{2}\right)\right.
$$

using a form of Fubini's theorem. In fact, first apply $x^{*} \in\left(L_{0}^{2}(P)\right)^{*}$ to both sides, then moving $x^{*}$ inside the integral which is permissible and since $x^{*} Z_{m}^{l}(\cdot)$ is a scalar measure, the classical Fubini theorem applies, cf. Dunford and Schwartz [3]. The structure function is obtained using the definition.

Thus the above representation can be extended for all locally isotropic random fields which need not be locally harmonizable. It is now possible to obtain an example of a structure function for a locally strongly harmonizable isotropic random field which is locally nonstationary. Thus consider the structure function $B(\cdot, \cdot, \cdot, \cdot)$, of a locally isotropic random field, by the previous theorem

$$
\begin{gathered}
B\left(\boldsymbol{s}, \boldsymbol{t}, \boldsymbol{s}+\boldsymbol{\tau}_{1}, \boldsymbol{t}+\boldsymbol{\tau}_{2}\right)=r_{1}\left(\left\|\boldsymbol{s}+\boldsymbol{\tau}_{1},\right\| \boldsymbol{t}+\boldsymbol{\tau}_{2} \|, \theta_{1}\right)+r_{2}\left(\left\|\boldsymbol{s}+\boldsymbol{\tau}_{1}\right\|,\|\boldsymbol{t}\|, \theta_{2}\right) \\
+r_{3}\left(\|\boldsymbol{s}\|,\left\|\boldsymbol{t}+\boldsymbol{\tau}_{2}\right\|, \theta_{3}\right)+r_{4}\left(\|\boldsymbol{s}\|,\|\boldsymbol{t}\|, \theta_{4}\right)+a \boldsymbol{\tau}_{1} \cdot \boldsymbol{\tau}_{2}
\end{gathered}
$$

where $r_{i}(\cdot, \cdot), i=1, \ldots, 4$ is of the form

$$
r_{i}\left(\|\boldsymbol{s}\|,\|\boldsymbol{t}\|, \theta_{i}\right)=\frac{1}{\omega_{n}} \sum_{m=0}^{\infty} \frac{h(m, n)}{C_{m}^{n}(1)} C_{m}^{n}\left(\cos \theta_{i}\right) b_{m}(\|\boldsymbol{s}\|,\|\boldsymbol{t}\|) .
$$

Suppose the spectral distribution function $F(\cdot, \cdot)$ is absolutely continuous, with density $f$, and has the specific form, which is clearly positive definite:

$$
f\left(\lambda, \lambda^{\prime}\right)=\frac{\omega_{n} \nu \lambda^{m+2 \nu+1}\left(\lambda^{\prime}\right)^{m+2 \nu+1}}{\alpha_{n}^{2}(m+\nu)} e^{\left(-\left(\lambda^{2}+\left(\lambda^{\prime}\right)^{2}\right)\right)}
$$

where as before, $\omega_{n}$ is the surface area of the unit sphere and $\nu=\frac{n-2}{2}$. Then it can be shown (see Swift [12]), that $r_{i}(\cdot, \cdot), i=1, \ldots, 4$, has the form

$$
r_{i}\left(\rho_{1}, \rho_{2}, \theta_{i}\right)=\left[1-2 \rho_{1}, \rho_{2} \cos \theta_{i}+\rho_{1}^{2} \rho_{2}^{2}\right]^{-\nu} e^{\left(-\left(\rho_{1}^{2}+\rho_{2}^{2}\right)\right)}
$$

where $\rho_{1}$ and $\rho_{2}$ are the specified lengths of the factors $\boldsymbol{s}, \boldsymbol{t}, \boldsymbol{s}+\boldsymbol{\tau}_{1}, \boldsymbol{t}+\boldsymbol{\tau}_{2}$, as given in the definition of $B(\cdot, \cdot, \cdot, \cdot)$. Thus, the structure function of a locally strongly harmonizable isotropic random field which is locally nonstationary is

$$
B\left(s, t, s+\tau_{1}, t+\tau_{2}\right)=\left[1-2\left\|s+\tau_{1}\right\|\left\|t+\tau_{2}\right\| \cos \theta_{1}+\left(\left\|s+\tau_{1}\right\|\left\|t+\tau_{2}\right\|\right)^{2}\right]^{-\nu}
$$




$$
\begin{gathered}
\times e^{\left(-\left(\left\|\boldsymbol{s}+\boldsymbol{\tau}_{1}\right\|^{2}+\left\|\boldsymbol{t}+\boldsymbol{\tau}_{2}\right\|^{2}\right)\right)} \\
+\left[1-2\left\|\boldsymbol{s}+\boldsymbol{\tau}_{1}\right\|\|\boldsymbol{t}\| \cos \theta_{2}+\left(\left\|\boldsymbol{s}+\boldsymbol{\tau}_{1}\right\|\|\boldsymbol{t}\|\right)^{2}\right]^{-\nu} e^{\left(-\left(\left\|\boldsymbol{s}+\boldsymbol{\tau}_{1}\right\|^{2}+\|\boldsymbol{t}\|^{2}\right)\right)} \\
+\left[1-2\|\boldsymbol{s}\|\left\|\boldsymbol{t}+\boldsymbol{\tau}_{2}\right\| \cos \theta_{3}+\left(\|\boldsymbol{s}\|\left\|\boldsymbol{t}+\boldsymbol{\tau}_{2}\right\|\right)^{2}\right]^{-\nu} e^{\left(-\left(\|\boldsymbol{s}\|^{2}+\left\|\boldsymbol{t}+\boldsymbol{\tau}_{2}\right\|^{2}\right)\right)} \\
+\left[1-2\|\boldsymbol{s}\|\|\boldsymbol{t}\| \cos \theta_{4}+(\|\boldsymbol{s}\|\|\boldsymbol{t}\|)^{2}\right]^{-\nu} e^{\left(-\left(\|\boldsymbol{s}\|^{2}+\|\boldsymbol{t}\|^{2}\right)\right)}+a \boldsymbol{\tau}_{1} \cdot \boldsymbol{\tau}_{2} .
\end{gathered}
$$

\section{Prediction for Local Fields}

Let $S_{r}$ denote a sphere in $\mathbb{R}^{n}$, of radius $r$, centered at the origin. Suppose that one observes $X(\boldsymbol{t})$, a locally harmonizable isotropic random field, at a countable number $\|\boldsymbol{t}\|=r_{i}, i \geq 1$ of points. These are some "instances", and could be taken as $0<r_{1}<r_{2}<\ldots$.

In this formulation, the problem is to obtain the best linear predictor in $L_{0}^{2}(P)$ of $X\left(t_{0}\right)$ for $\|\boldsymbol{t}\|=r\left(\neq r_{i}, i \geq 1\right)$. In the following work, it is assumed that the tempered spectral bimeasure, $F(\cdot, \cdot)$ of $X(\cdot)$ is strictly positive definite.

Rao [10] solved the linear prediction problem for harmonizable isotropic fields, the work in this section is an extension of his work to the local case.

Let $M=\overline{s p}\left\{X(\boldsymbol{t}):\|\boldsymbol{t}\|=r_{i}, i \geq 1\right\}$, then $M \subset L_{0}^{2}(P)$ and for $\left\|\boldsymbol{t}_{0}\right\|=r>0, X\left(\boldsymbol{t}_{0}\right) \notin M$. The linear prediction problem is to find a unique $Y$ in $M$ such that

$$
\left\|X\left(t_{0}\right)-Y\right\|_{2}=\inf \left\{\left\|X\left(t_{0}\right)-W\right\|_{2}: W \in M\right\} .
$$

Using a classical result of $\mathrm{F}$. Riesz, there is a unique $Y$ in $M$ satisfying

$$
\left(X\left(t_{0}-Y\right)\right) \perp M
$$

That is, $Y$ is a solution of the infinite system of linear equations

$$
E\left(X\left(\boldsymbol{t}_{0}\right) \overline{X(\boldsymbol{t}))}=E\left(Y \overline{X(\boldsymbol{t}))} \text { for all }\|\boldsymbol{t}\|=r_{i}, \quad i \geq 1 .\right.\right.
$$

This produces the desired solution once a representation of elements of $M$ is obtained. This representation can be obtained from an isometric identification of $M$ with an appropriate sequence space $\ell_{F}^{2}$ with respect to a positive definite weight function $F(\cdot, \cdot)$. This is given by:

Proposition 5.1: Each element $Y$ of $M$, the subspace of $L_{0}^{2}(P)$ introduced above is representable as:

$$
Y=\sum_{k=1}^{\infty} \sum_{m=0}^{\infty} \sum_{l=1}^{h(m, n)} a(l, m, k) v(k, t)
$$

where

$$
v(k, t)=\int_{+0}^{\infty} \frac{J_{m+\nu}(\lambda\|\boldsymbol{t}\|)}{(\lambda\|\boldsymbol{t}\|)^{\nu}} d Z_{m}^{l}\left(\lambda_{k}\right)+\boldsymbol{X}_{1} \cdot \boldsymbol{t}
$$

and $Z_{m}^{l}(\cdot)$ is the stochastic measure representing the random field $X(\cdot)$, and the sequence $\{a(l, m, k): m \geq 0,1 \leq l \leq h(m, n), k \geq 1\}$ satisfies the condition:

$$
0 \leq \sum_{n=0}^{\infty} \sum_{l=1}^{h(m, n)} \int_{+0}^{\infty} \int_{+0}^{\infty} \sum_{k=1}^{\infty} \sum_{k^{\prime}=1}^{\infty} a(l, m, k) \overline{a\left(l, m, k^{\prime}\right)} v\left(k, \boldsymbol{s}_{k}\right) \overline{v\left(k^{\prime}, \boldsymbol{t}_{k^{\prime}}\right)}<\infty
$$


with $E\left(Z_{m}^{l}(A) \bar{Z}_{m^{\prime}}^{l^{\prime}}(B)\right)=F(A, B) \delta_{m m^{\prime}} \delta_{l l^{\prime}}$ and $\nu=\frac{n-2}{2}$.

Proof: The proof of this proposition follows from standard arguments and is similar to that of Rao [10]. The details are outlined here. Let $\ell_{J_{\nu}, F}$ be the vector space of scalar sequences $a=$ $\{a(l, m, k): m \geq 0,1 \leq l \leq h(m, n), k \geq 1\}$ such that $\|\boldsymbol{a}\|_{2, J_{\nu, F}^{2}}$ is given by

$$
0 \leq \alpha_{n}^{2} \sum_{m=0}^{\infty} \sum_{l=1}^{h(m, n)} \int_{+0}^{\infty} \int_{+0}^{\infty} \sum_{k=1}^{\infty} \sum_{k^{\prime}=1}^{\infty} a(l, m, k) \overline{a\left(l, m, k^{\prime}\right)} v(k, s) \overline{v\left(k^{\prime}, t\right)}<\infty
$$

so that $\|\cdot\|_{2, J_{\nu}, F}$ is a norm. For $\boldsymbol{a}, \boldsymbol{b} \in \ell_{J_{\nu}, F}$ the inner product is defined by the same expression with $\overline{a(l, m, k)}$ replaced with $\overline{b(l, m, k)}$. Using this, it follows that $\left(\ell_{J_{\nu}, F},\|\cdot\|_{2, J_{\nu}, F}\right)$ is a Hilbert sequence space and taking $a(l, m, k)=S_{m}^{l}(\boldsymbol{u})$, the spherical harmonic, for $k=\left\|\boldsymbol{t}_{k}\right\|$ and $=0$ for $k \neq\left\|\boldsymbol{t}_{k}\right\|$, one gets $i: X(\boldsymbol{t}) \rightarrow \boldsymbol{a}$ with $\boldsymbol{t}=(\|\boldsymbol{t}\|, \boldsymbol{u})$ which satisfies $E\left(|X(\boldsymbol{t})|^{2}\right)=$ $\|\boldsymbol{a}\|_{2, J_{\nu}, F}^{2}=E\left(|i X(\boldsymbol{t})|^{2}\right)$. Thus $i$ is an isometry and can be extended by linearity and polarization to all of $M$ onto $\ell_{J_{\nu}, F}$. It thus follows from standard arguments that there is a unique $\boldsymbol{a} \in \ell_{J_{\nu}, F}$ so that $Y=i \boldsymbol{a}$, but this is the desired result in explicit form.

Let $Y_{0} \in M$ be the unique element that is closest to $X\left(t_{0}\right)$. Since $Y_{0}$ satisfies a system of equations as mentioned above, the desired solution is obtained if the vector $\boldsymbol{a}$ is found such that

$$
E\left(X\left(t_{0}\right) \overline{X(t))}=E\left(Y_{0} \overline{X(t))} .\right.\right.
$$

Using the proposition, along with the spectral representation of $X(\cdot)$ in spherical-polar coordinates, one multiplies by $S_{m}^{l}(\boldsymbol{v})$ then integrates over $S_{n}$, the unit sphere in $\mathbb{R}^{n}$, which gives

$$
\begin{gathered}
\sum_{k=1}^{\infty} a(l, m, k)\left(\frac{\|\boldsymbol{t}\|}{\left\|\boldsymbol{t}_{k}\right\|}\right)^{\nu} \int_{+0}^{\infty} \int_{+0}^{\infty} \frac{J_{m+\nu}\left(\lambda\left\|\boldsymbol{t}_{k}\right\|\right) J_{m+\nu}\left(\lambda^{\prime}\left\|\boldsymbol{t}_{l}\right\|\right)}{\left(\lambda \lambda^{\prime}\right)^{\nu}} d F\left(\lambda, \lambda^{\prime}\right) \\
=\alpha_{n} S_{m}^{l}(\boldsymbol{u}) \int_{+0}^{\infty} \int_{+0}^{\infty} \frac{J_{m+\nu}(\lambda\|\boldsymbol{t}\|) J_{m+\nu}\left(\lambda^{\prime}\left\|\boldsymbol{t}_{l}\right\|\right)}{\left(\lambda \lambda^{\prime}\right)^{\nu}} d F\left(\lambda, \lambda^{\prime}\right)
\end{gathered}
$$

by the orthonormality of the $S_{m}^{l}(\boldsymbol{v})$ relative to the surface measure on $S_{n}$. Further simplification gives:

Theorem 5.1: Let $\left\{X\left(\left\|\boldsymbol{t}_{k}\right\|, \boldsymbol{u}_{k}\right): k=1,2, \ldots\right\}$ be an observed sequence of a locally weakly harmonizable isotropic random field $X: \mathbb{R}^{n} \rightarrow L_{0}^{2}(P)$. Then the best linear least squares predicator $Y_{0}$ of $X\left(\boldsymbol{t}_{0}\right), \boldsymbol{t}_{0}=\left(\left\|\boldsymbol{t}_{0}\right\|, \boldsymbol{u}_{0}\right), 0<\left\|\boldsymbol{t}_{0}\right\| \neq\left\|\boldsymbol{t}_{k}\right\|, k=1,2, \ldots$ is given by

$$
Y=\sum_{k=1}^{\infty} \sum_{m=0}^{\infty} \sum_{l=1}^{h(m, n)} a(l, m, k) v\left(k, t_{k}\right)
$$

with coefficients

$$
\boldsymbol{a}=\{a(l, m, k): 1 \leq l \leq h(m, n), m \geq 0, k \geq 1\}
$$

as a unique solution to the equation:

$$
\sum_{k=1}^{\infty} a(l, m, k)\left(\frac{\|\boldsymbol{t}\|}{\left\|\boldsymbol{t}_{k}\right\|}\right)^{\nu} \int_{+0}^{\infty} \int_{+0}^{\infty} \frac{J_{m+\nu}\left(\lambda\left\|\boldsymbol{t}_{k}\right\|\right) J_{m+\nu}\left(\lambda^{\prime}\left\|\boldsymbol{t}_{l}\right\|\right)}{\left(\lambda \lambda^{\prime}\right)^{\nu}} d F\left(\lambda, \lambda^{\prime}\right)
$$




$$
=\alpha_{n} S_{m}^{l}(\boldsymbol{u}) \int_{+0}^{\infty} \int_{+0}^{\infty} \frac{J_{\mu+\nu}(\lambda\|\boldsymbol{t}\|) J_{m+\nu}\left(\lambda^{\prime}\left\|\boldsymbol{t}_{l}\right\|\right)}{\left(\lambda \lambda^{\prime}\right)^{\nu}} d F\left(\lambda, \lambda^{\prime}\right) .
$$

\section{Acknowledgements}

The author expresses his thanks to Professor M.M. Rao for his advice and encouragement during the work of this project. The author also expresses his gratitude to the Mathematics Department at Western Kentucky University for release time during the Spring 1994 semester, during which this work was completed.

\section{References}

[1] Adler, R.J., The Geometry of Random Fields, John Wiley, New York 1981.

[2] Chang, D.K. and Rao, M.M., Bimeasures and nonstationary processes, In: Real and Stochastic Analysis, John Wiley, New York (1986), 7-118.

[3] Dunford, N. and Schwartz, J.T., Linear Operators, Part I, Interscience, New York 1957.

[4] Gelfand, I.M. and Vilenkin, N.Ya., Generalized Functions, Vol. 1, Appl. of Harmonic Analysis, Academic Press, New York 1964.

[5] Gihman, I.I. and Skorohod, A.V., The Theory of Stochastic Processes I, Springer-Verlag, New York 1974.

[6] Lebedev, N.N., Special Functions and Their Applications, Dover Publications, Inc., New York 1972.

[7] Müller, C., Sperical Harmonics, Lecture Notes in Mathematics, Vol. 17, Springer-Verlag, New York 1966.

[8] Rao, M.M., Harmonizable processes: Structure theory, L'Enseign Math. 28 (1982), 295351.

[9] Rao, M.M., Representation theory of multidimensional generalized random fields, $J$. Multiv. Anal. 2 (1969), 411-435.

[10] Rao, M.M., Sampling and prediction for harmonizable isotropic random fields, J. Combin. Info. Sys. Sci. 16:2-3 (1991), 207-220.

[11] Ryshik, I.M. and Gradstein, I.S., Tables of Series, Products and Integrals, VEB Deutscher Verlag der Wissenschaften, Berlin 1963.

[12] Swift, R., The structure of harmonizable isotropic random fields, Stoch. Anal. Appl. 12:5 (1994), 583-616.

[13] Swift, R., A class of harmonizable isotropic random fields, J. Combin. Info. Sys. Sci., (to appear).

[14] Swift, R., Stochastic processes with harmonizable increments, preprint.

[15] Watson, G.N., A Treatise on the Theory of Bessel Functions, Cambridge University Press, London 1962.

[16] Yadrenko, M.I., Spectral Theory of Random Fields, Optimization Software, Inc., New York (English translation) 1983.

[17] Yaglom, A.M., Correlation Theory of Stationary and Related Random Functions, Vols. 1 and 2, Springer-Verlag, New York 1987.

[18] Yaglom, A.M., Some classes of random fields in $n$-dimensional space related to stationary random processes, Theory Prob. Appl. 2:3 (1957), 273-320. 


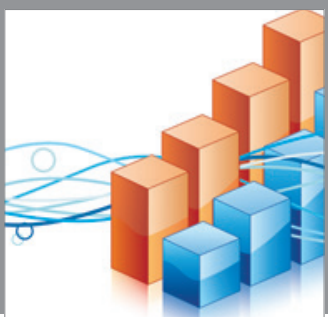

Advances in

Operations Research

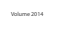

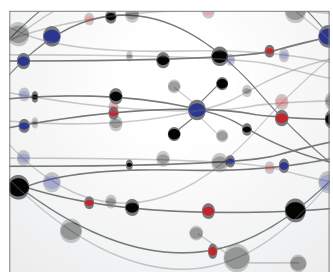

\section{The Scientific} World Journal
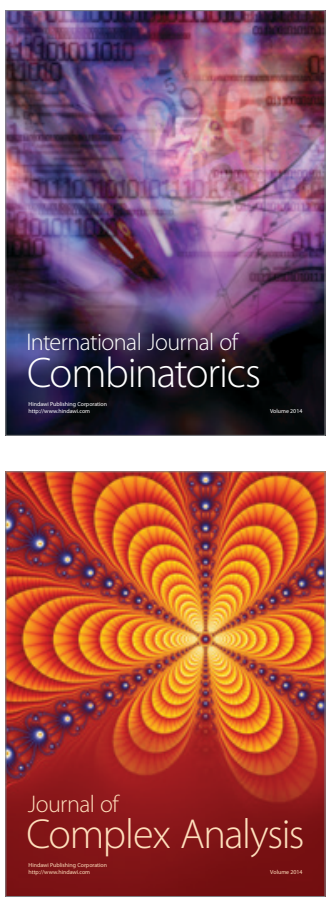

International Journal of

Mathematics and

Mathematical

Sciences
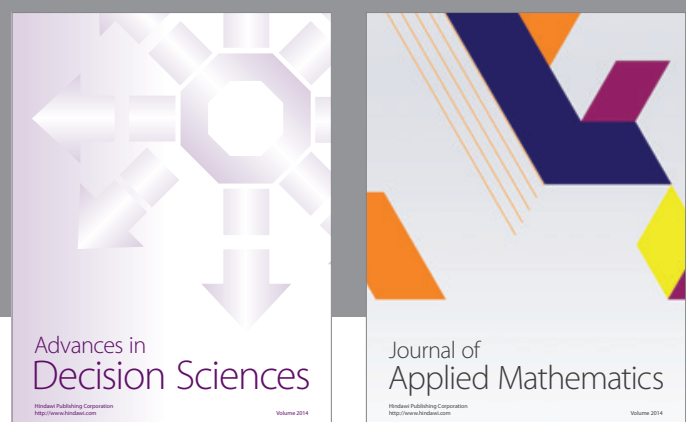

Journal of

Applied Mathematics
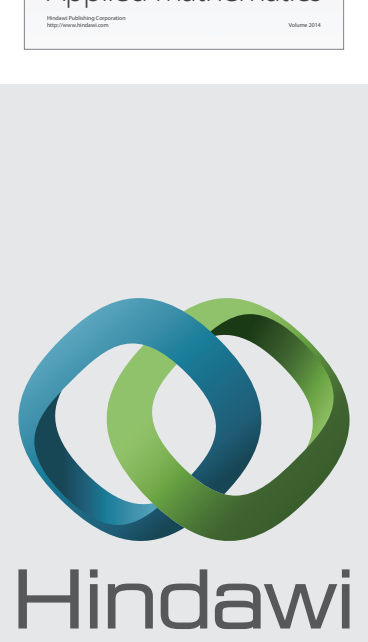

Submit your manuscripts at http://www.hindawi.com
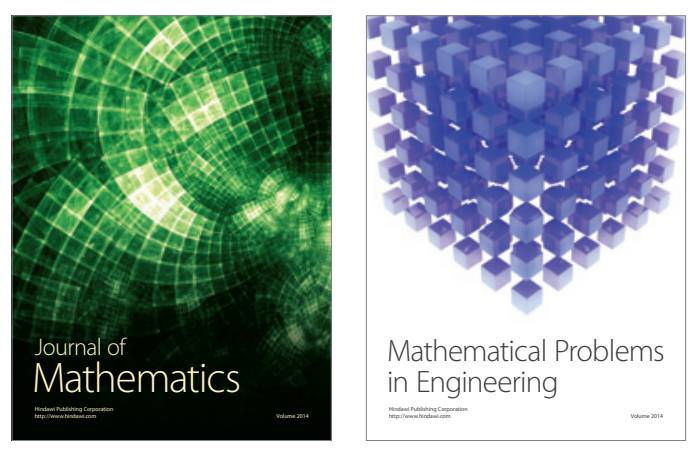

Mathematical Problems in Engineering
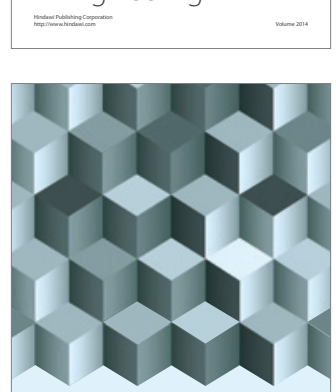

Journal of

Function Spaces
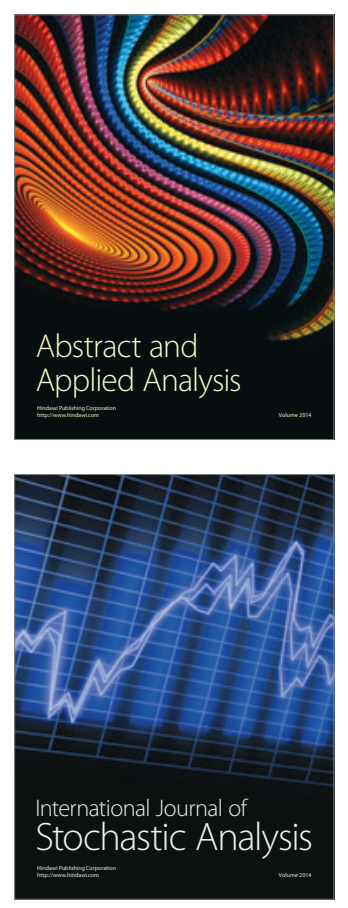

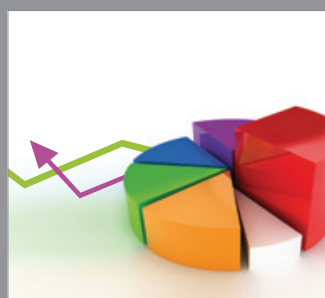

ournal of

Probability and Statistics

Promensencen
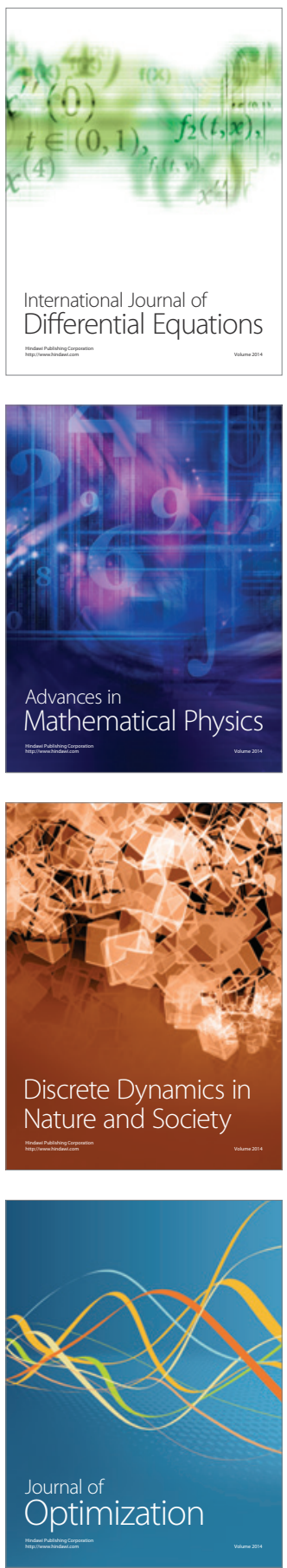